JGG 2020;68:1-7

doi: 10.36150/2499-6564-356

\title{
Atherosclerosis impacts the link between hepatocyte growth factor and cognition
}

\author{
Nermien N. Adly ${ }^{1}$, Wessam H. El-kawaly ${ }^{1}$, Hoda A. Abdelsattar ${ }^{2}$ \\ ${ }^{1}$ Geriatrics and Gerontology department, Faculty of Medicine, Ain Shams University, Cairo, \\ Egypt; ${ }^{2}$ Clinical pathology department, Faculty of Medicine, Ain Shams University, Cairo, Egypt
}

Received: July 9, 2019

Accepted: October 9, 2019

\section{Correspondence \\ Nermien N. Adly \\ Geriatrics and Gerontology Department, Faculty of Medicine, Ain Shams University, Abbassia, Cairo, Egypt \\ Tel. +00201114101969 \\ E- mail: drnermienadly@yahoo.com}

\section{Conflict of interest}

The Authors declare no conflict of interest

How to cite this article: Adly NN, Elkawaly $\mathrm{WH}$, Abdelsattar HA. Atherosclerosis impacts the link between hepatocyte growth factor and cognition. Journal of Gerontology and Geriatrics 2020;68:1-7. https://doi.org/10.36150/2499-6564-356

C Copyright by Società Italiana di Gerontologia e Geriatria (SIGG)

\section{(c) (i) $(9)$}

\section{OPEN ACCESS}

This is an open access article distributed in accordance with the CC-BY-NC-ND (Creative Commons Attribution-NonCommercial-NoDerivatives 4.0 International) license. The article can be used by giving appropriate credit and mentioning the license, but only for non-commercial purposes and only in the original version. For further information: https://creativecommons.org/licenses/by-nc-nd/4.0/deed.en
Background. There is a controversy about the association between Hepatocyte growth factor (HGF) and cognition. Increased serum level of HGF has been reported in patients with hypertension, peripheral arteriosclerosis and carotid atherosclerosis. Ankle-brachial index $(\mathrm{ABI})$ is considered as a marker of atherosclerosis. we hypothesized that hypertension or atherosclerosis with hypertension could alter the relation between HGF serum level and cognitive function.

Aim. To study HGF and cognitive function in hypertensives with and without atherosclerosis versus healthy controls.

Methods. This case-control study included ninety elderly subjects attending outpatient primary care geriatric clinics. They were subdivided into 3 groups; Group A (30 normotensives with normal Ankle-brachial index $(\mathrm{ABI})$ as controls, group $B$ (30 hypertensives with normal $A B I)$ and group $\mathrm{C}$ (30 hypertensives with abnormal $\mathrm{ABI}$ ) as cases. Cognitive function was assessed by Rowland Universal Dementia Assessment Scale (RUDAS).

Results. Group $C$ had worse score in RUDAS than controls $(P=0.01)$. HGF was negatively correlated with $A B I$ in group $C(p=0.007)$. HGF was positively associated with RUDAS score, in group $A(p<0.001)$, in group $B$; after further adjustment for systolic blood pressure (SBP) $(p=0.024)$ and in group $C$; after adjustment for $A B I(p=0.031)$ or $A B I$ and SBP $(p=0.05)$.

Conclusions. The potential beneficial link between HGF serum concentration and cognition was met in normotensive subjects with normal $A B I$. However, this link is halted in the presence of hypertension or atherosclerosis as assessed by ABI. Alternatively, the raised HGF serum level may be an epiphenomenon of atherosclerosis.

Key words: ankle-brachial index, atherosclerosis, cognition, hepatocyte growth factor, hypertension

\section{INTRODUCTION}

Hepatocyte growth factor (HGF) is a heparin-binding polypeptide (728 amino acids) that regulates the growth, migration, and morphogenesis of various cells ${ }^{1}$. An increased serum level of HGF has been reported in patients with hypertension, peripheral arteriosclerosis, carotid atherosclerosis, and coronary artery disease ${ }^{1,2}$.

HGF has been shown to have an anti-apoptotic action on the endothelium ${ }^{3}$, it has been detected in atherosclerotic plaques ${ }^{4}$, and it shows a positive association with atherosclerosis ${ }^{5}$. 
Atherosclerotic vascular disease affects large- and medium-sized arteries of most circulatory beds and it is the leading cause of death and disability in developed countries. Lower-extremity atherosclerosis, peripheral arterial disease (PAD), is a significant public health problem ${ }^{6}$.

There is a controversy about the association between HGF serum level and cognition. Although some consider HGF as a new targeted therapy in dementia ${ }^{7}$, others found that it is associated with cognitive dysfunction ${ }^{8}$, with reports about increased HGF levels in the cerebrospinal fluid (CSF) of patients with $A D{ }^{9}$. Similarly, others found that HGF serum level is associated with the presence of cardiovascular disease (CVD) risk factors ${ }^{10}$. Recently, literature has increased interest in evaluating the link between dementia and serum HGF ${ }^{11}$. ZhuY et al found that magnetic resonance imaging (MRI) markers of small vessel disease (SVD) rather than large vessel disease were associated with higher serum HGF, among those with cognitive impairment not demented or Alzheimer's disease ${ }^{11}$.

As previous literature reported the presence of SVD in normal elderly ${ }^{12}$ and diabetics ${ }^{13}$, along with the supposed cross talk between large and small arteries in hypertensives ${ }^{14}$, the current work aimed to explore the link between HGF serum level and cognition in hypertensive elderly with abnormal ankle-brachial index $(\mathrm{ABI})$, as a known marker of atherosclerosis ${ }^{15,16}$, and hypertensive elderly with normal $\mathrm{ABI}$ versus controls (normotensives with normal $\mathrm{ABI}$ ), among non-diabetic subjects.

As atherosclerosis, which is of a major link to hypertension ${ }^{17}$, is linked to vascular dementia ${ }^{8}$, we hypothesized that hypertension or atherosclerosis with hypertension could alter the relation between HGF serum level and cognitive function.

\section{MATERIALS AND METHODS}

A case-control study was conducted among elderly subjects, aged $\geq 60$ years ${ }^{18,19}$, attending outpatient primary care geriatric clinic. Ninety eligible elderly patients were consecutively included and were subdivided into 3 groups. Group A included 30 normotensive subjects with normal $A B I$ as controls, group $B$ included 30 hypertensive subjects with normal $A B I$ as cases and group $C$ included 30 hypertensive subjects with abnormal $\mathrm{ABI}$ as cases. The data were collected from January 2017 to June 2017.

Those with diabetes mellitus, severe liver or renal dysfunction or cancer were excluded from the study because serum levels of HGF are well known to be influenced by these diseases ${ }^{20,21}$.
Subjects were excluded if they had severe sensory or cognitive impairment which could interfere with the assessment or refused to participate.

The minimum sample size was based upon the odd ratio for the association between $\mathrm{ABI}$ and cognitive test performance in elderly ${ }^{22}$. Using Epi-info program, version 3.5.1, power 1-ß 80\%, and confidence interval of $95 \%$, the minimum number was 16 for cases and 16 for controls.

\section{Cognitive function}

It was assessed by The Rowland Universal Dementia Assessment Scale (RUDAS). The RUDAS is a cognitive assessment tool that was created for culturally and linguistically diverse populations. The RUDAS is a 6-item questionnaire that assesses multiple cognitive domains and can be administered in less than 10 minutes. In the original validation study of RUDAS, both the interrater and test-retest reliabilities of the test were very high. Compared with the criteria of the Diagnostic and Statistical Manual of Mental Disorders, 4th edition (DSM-IV), the RUDAS was found to have a sensitivity of $89 \%$ and a specificity of $98 \%$. Performance on the RUDAS was not affected by years of education or the preferred language ${ }^{23}$.

\section{DIAGNOSIS OF HYPERTENSION}

Blood pressure was measured using a standard mercury sphygmomanometer in the right arm, in supine position after rest for 5 minutes. Systolic and diastolic blood pressure was recorded as the mean of two measurements. The diagnosis of hypertension was based upon known history of hypertension diagnosis and its treatment or it was based upon the cutoff values stated by the National heart foundation of Australia, systolic $\geq 140 \mathrm{mmhg}$ and/ or diastolic $\geq 90 \mathrm{mmhg}$, on two separate occasions, at least one week apart ${ }^{24}$.

\section{Measurement of ANKLE-BRACHial INDEX}

The $A B I$ is an objective non-invasive reproducible measure that reflects $P A D$ severity ${ }^{15}$, and $A B I$ is considered as a marker of atherosclerosis in the Cardiovascular Health Study, Cardiovascular Heart Study Collaborative Research Group ${ }^{16}$.

$\mathrm{ABI}$ was assessed by hand-held vascular Doppler, BT200, HI-dop. Steps were applied according to known references, with abnormal $\mathrm{ABI}<0.9^{11,25}$.

\section{LABORATORY DATA}

\section{SAMPLING}

Five milliliters of fasting venous blood were collected under complete aseptic precautions in plain test tubes. 
The serum was separated by centrifugation (1000x $\mathrm{g}$ for 15 minutes) and was divided into two aliquots. One was designated for the immediate assay of fasting lipid profile. The other aliquot was stored at $-20^{\circ} \mathrm{C}$ for subsequent assay of HGF. Hemolysed samples were discarded. Repeated freezing and thawing were avoided.

\section{ANALYTICAL METHODS}

Serum fasting lipid profile [total cholesterol, triglycerides (TG) and high-density lipoprotein- cholesterol (HDL-C)] was measured using Synchron CX-9 autoanalyser (Beckman Instruments Inc.; Scientific Instruments Division, Fillerton, CA 92634, 3100, USA). Low density lipoprotein- cholesterol (LDL-C) value was calculated according to Friedewald equation. HGF assay was done using the commercially available enzymelinked immunosorbent assay (ELISA) kit, supplied by Elabscience Company (Building 4, Room 401, Guandong Science and Technology Industry Park, Wuhan, P.R.C.).

\section{STATISTICAL METHOD}

SPSS version 16 (SPSS Inc., Chicago, IL, USA) was used for data analysis. Qualitative data were expressed in the form of number and percentage and were compared using Chi-Square test. Quantitative data were expressed in the form of mean \pm SD for parametric data or median and interquartile range for non-parametric data and were compared using ANOVA test (with least significant difference as post-hoc test), after log transformation of non-parametric data.

Distribution normality was assessed using a $z$ test for skewness ${ }^{26}$.

Generalized linear model was used to study the significance of serum HGF level as a predictor of cognitive function, linear distributional assumption was used, in each group, after adjustment for the possible confounding variables.

\section{RESULTS}

Using ANOVA, there was no significant difference between the 3 groups in age $(p=0.98)$.

Serum HGF concentration was higher in group C (hypertensives with abnormal $\mathrm{ABI}$ ) compared with group $A$ (controls) or $B$ (hypertensives with normal $A B I$ ) ( $p<0.001$ and 0.025 consecutively), and serum HGF concentration was higher in group $B$ than group $A$ $(p=0.028)(T a b . ~ I)$.

Group $C$ had worse RUDAS score than controls ( $P=$ 0.01), with no significant difference between group $B$ and $\mathrm{C}$ in RUDAS score $(\mathrm{p}=0.22)$ (Tab. I).

Table I. Comparing characteristic data between the 3 groups ${ }^{18}$.

\begin{tabular}{|c|c|c|c|c|c|c|c|}
\hline & Group A (controls) & $\begin{array}{c}\text { Group B } \\
\text { (hypertensives with } \\
\text { normal ABI) }\end{array}$ & \begin{tabular}{|c|} 
Group C \\
(hypertensives with \\
abnormal ABI)
\end{tabular} & P-value & P1 & P2 & P3 \\
\hline Age (years) & $69.20 \pm 10.31$ & $69.23 \pm 7.04$ & $69.60 \pm 7.28$ & 0.98 & & & \\
\hline Males (n, \%) & $24(80 \%)$ & $14(46.7 \%)$ & $16(53.3 \%)$ & 0.02 & & & \\
\hline Education years* & $0(0-6)$ & $0(0-5.5)$ & $0(0-6)$ & 0.84 & & & \\
\hline Smoking Index* & $130(0-142.5)$ & $0(0-60)$ & $0(0-137.5)$ & 0.31 & & & \\
\hline IHD & $7(25.0 \%)$ & $9(30.0 \%)$ & $25(83.3 \%)$ & $<0.001$ & & & \\
\hline Stroke & $1(3.6 \%)$ & $2(6.7 \%)$ & $3(10.0 \%)$ & 0.62 & & & \\
\hline SBP (mmhg) & $123.3 \pm 11.24$ & $134.7 \pm 16.3$ & $132.7 \pm 12.9$ & 0.004 & 0.002 & 0.01 & 0.57 \\
\hline DBP (mmhg) & $77.67 \pm 6.79$ & $82.33 \pm 9.32$ & $81 \pm 8.9$ & 0.092 & & & \\
\hline $\mathrm{BMI}^{*}$ & $24(21.7-26.75)$ & $29.4(25.4-32)$ & $26.6(23.6-36.7)$ & $<0.001$ & 0.001 & 0.001 & 0.99 \\
\hline WC (cm) & $96 \pm 17.18$ & $112.6 \pm 3.8$ & $109.2 \pm 21.19$ & 0.011 & 0.006 & 0.02 & 0.59 \\
\hline GDS-15 score* & $2(1.75-3)$ & $2(0-3)$ & $2(1-4.75)$ & 0.14 & & & \\
\hline T chol. (mg/dl) & $137 \pm 47.7$ & $186.6 \pm 59.7$ & $164.1 \pm 50.8$ & 0.003 & 0.001 & 0.051 & 0.11 \\
\hline TG (mg/dl) & $114.9 \pm 50.2$ & $146.9 \pm 54.5$ & $149.1 \pm 57.9$ & 0.03 & 0.033 & 0.02 & 0.88 \\
\hline LDL-C (mg/dl) & $87.1 \pm 36.8$ & $122 \pm 36.6$ & $104.9 \pm 39.9$ & 0.004 & 0.001 & 0.08 & 0.09 \\
\hline $\mathrm{HDL}-\mathrm{C}(\mathrm{mg} / \mathrm{dl})$ & $37.8 \pm 12.43$ & $43.5 \pm 17.37$ & $37.79 \pm 11.02$ & 0.22 & & & \\
\hline$\left.A B\right|^{\star}$ & $0.93(0.91-0.97)$ & $0.93(0.92-0.93)$ & $0.6(0.5-0.7)$ & $<0.001$ & 0.82 & $<0.001$ & $<0.001$ \\
\hline RUDAS score & $27.13 \pm 2.78$ & $26.13 \pm 3.05$ & $25.2 \pm 3.30$ & 0.049 & 0.21 & 0.01 & 0.22 \\
\hline HGF $(p g / m l)^{*}$ & $170(100-350)$ & $345(130-838)$ & $795(250-1343.8)$ & $<0.001$ & 0.028 & $<0.001$ & 0.025 \\
\hline
\end{tabular}

ABI: Ankle-Brachial Index; BMI: Body Mass Index; DBP: Diastolic Blood Pressure; GDS-15: Geriatric Depression Scale-15 items; HDL-C: High Density Lipoproteincholesterol; HGF: Hepatocyte Growth Factor; HHD: ischemic heart disease; LDL-C: Low Density Lipoprotein-cholesterol; RUDAS: Rowland Universal Dementia Assessment SCALE, SBP: Systolic Blood Pressure; T chol.: Total cholesterol; TG: Triglycerides; WC: waist circumference; *: data were expressed as median (interquartile range) for non-parametric quantitative data; P1, P2 and P3: P values for post-hoc analysis; P1: Group A vs Group B; P2: Group A vs Group C; P3: Group B vs Group C. 
By Pearson correlation, serum HGF concentration was positively correlated with RUDAS score only in group $A$ $(p=0.035, r=0.4)$. HGF concentration was negatively correlated with $\mathrm{ABI}$, in group $\mathrm{C}(\mathrm{p}=0.007, r=-0.49)$, and it was positively correlated with systolic blood pressure (SBP), in group $B(p=0.045, r=0.37)$. In group C, HGF was positively correlated with SBP, after adjustment for $\mathrm{ABI}$. Serum HGF concentration was not correlated with lipid profile.

Generalized linear model, in each group, revealed the followings; in group A, serum HGF concentration was positively associated with RUDAS score $(p<0.001)$. In group $B$, serum HGF concentration was positively associated with RUDAS score only after further adjustment for SBP $(p=0.024)$. In group $C$, serum HGF concentration was positively associated with RUDAS score only after adjustment for $\mathrm{ABI}$ or $\mathrm{ABI}$ and SBP ( $p=0.031$ and 0.05 consecutively) (Tab. II).

After further adjustment for lipid profile, the significant association between HGF and RUDAS score was found only in controls $(p=0.006$, $O R=1.004$ and $\mathrm{Cl}=1.001$ 1.006).

Furthermore, adjustment for gender, body mass index, ischemic heart disease and lipids doesn't affect the significant association between HGF and RUDAS score in controls $(p=0.017$, OR $=1.004$ and $\mathrm{Cl}=1.001$ 1.006).

\section{DISCUSSION}

The current data explored that HGF was higher in hypertensives with/or without abnormal $A B \mid$ than controls, and hypertensives with abnormal $A B I$ had higher HGF than hypertensives with normal $A B I$. Therefore, HGF might mediate the pathology of hypertension \pm atherosclerosis. Alternatively, the raised HGF serum level may be an epiphenomenon of hypertension \pm atherosclerosis.

This is in accordance with Yoshitomi et al. ${ }^{27}$ who found that patients with PAD showed higher serum HGF concentrations than controls.

Similarly, Nakamura et al. ${ }^{28}$ explored that serum HGF concentration in hypertensive subjects without any complication was higher than normotensive subjects ( $p<0.001)$. Furthermore, serum HGF concentration in hypertensive patients with complications was significantly higher than those without complication or normotensive subjects.

Recently, Bell et al. ${ }^{29}$ proved the positive association

Table II. Predictors/associates of RUDAS score, in each group.

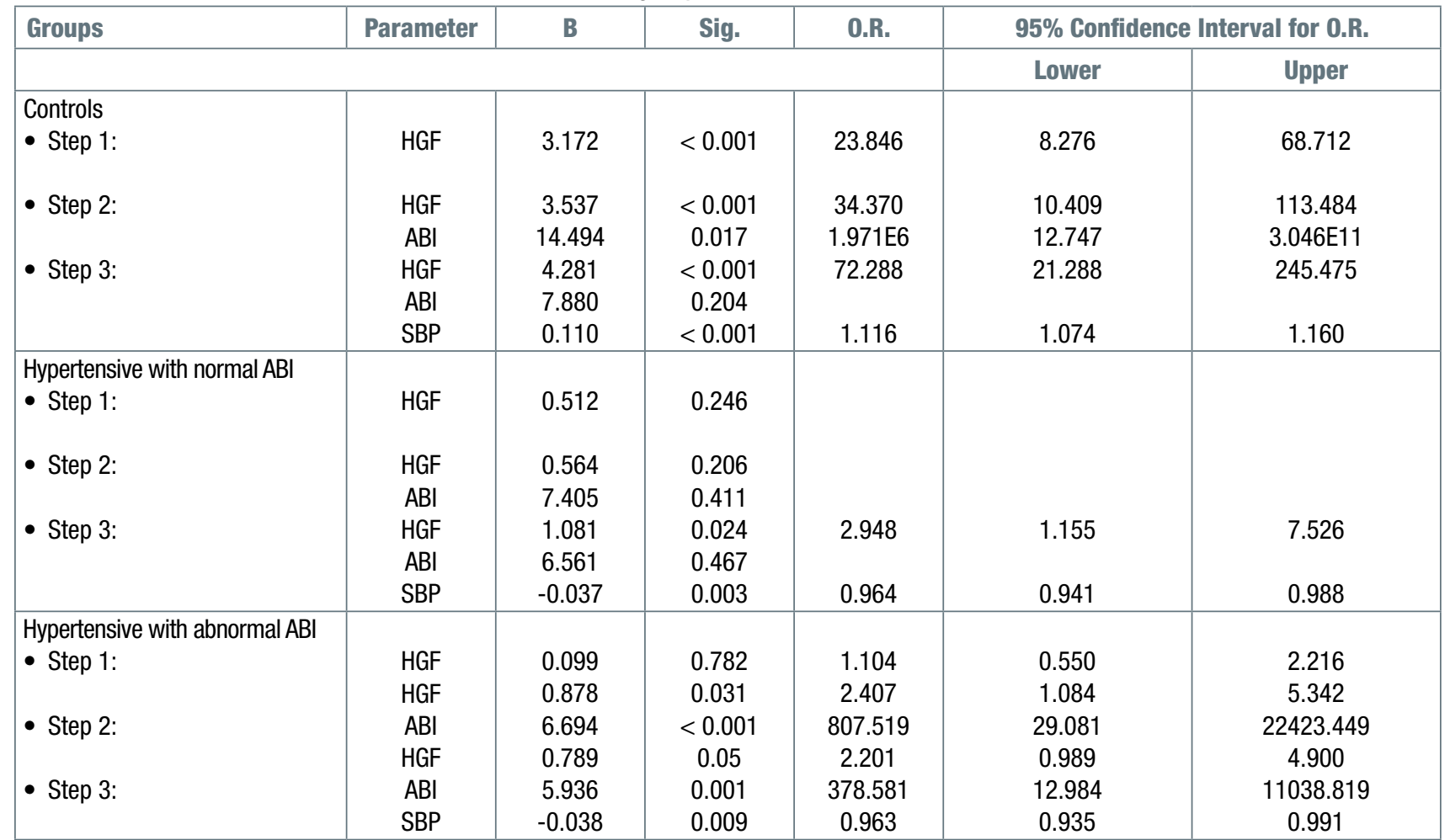

ABI: Ankle-Brachial Index; HGF: Hepatocyte Growth Factor; RUDAS: Rowland Universal Dementia Assessment Scale; SBP: Systolic Blood Pressure; step 1: HGF was used in regression alone; step 2: adjusted regression for $\mathrm{ABl}$; step 3: adjusted regression for $\mathrm{ABI}$ and systolic blood pressure. 
between higher circulating HGF levels at baseline and the progression of atherosclerosis, as defined by coronary artery calcium and carotid plaques, among participants aged 45-84 years. Additionally, Decker et al. ${ }^{30}$ reported positive relation between changes in HGF levels and clinical coronary heart disease; rather than subclinical atherosclerosis, as defined by coronary artery calcium.

The positive association between SBP and HGF serum level, in the current work, was in accordance with Nakamura et al. ${ }^{28}$.

The current results explored that serum HGF concentration was negatively associated with $A B I$, as a marker of atherosclerosis ${ }^{15,16}$. This might be explained by Taher et al. ${ }^{4}$ who declared that the migration of vascular smooth muscle cells (VSMCs) to the intima is the main event in neo-intima formation and atherogenesis. They found that Met, the receptor for HGF, is expressed on VSMCs derived from the intima of atherosclerotic plaques. Furthermore, HGF promoted VSMC migration across fibronectin-coated filters. Their findings suggested a role for the HGF in the pathogenesis of atherosclerosis and restenosis.

Although some previous evidence found a possible role for serum HGF concentration for the treatment of $P A D$, through angiogenesis and improved necrosis in rat ischemic limbs ${ }^{31}$, these positive results were not the same for humans in two multicenter, double blind, placebo controlled clinical trials by Powell et al. ${ }^{32}$ and Shigematsu et al. ${ }^{33}$.

In the current study, serum HGF concentration was positively associated with cognitive performance, in controls. These data are supported by previous experimental studies which revealed improvement in neurodegenerative diseases by $\mathrm{HGF}^{34,35}$.

The beneficial effect of HGF could be explained by Takeuchi et al who found that injection of A-beta peptide decreases the blood-vessel density which improved after HGF expression ${ }^{36}$. A decrease in cerebral blood flow is observed in Alzheimer disease patients ${ }^{37}$.

These findings are also attributed to the anti-apoptotic effect of HGF on endothelium via Bcl-2 induction, Bcl-2 is an inhibitor of apoptosis ${ }^{3}$.

On the other hand, previous studies found increased HGF levels in the CSF of patients with $A D{ }^{9}$, however other cardiovascular risk factors were not considered. Recently, ZhuY et al. linked MRI markers of SVD, rather than large vessel disease, to higher serum HGF levels, among those with cognitive impairment not demented or Alzheimer's disease subjects, even after controlling for cardiovascular risk factors ${ }^{11}$. ZhuY et al. excluded vascular dementia and stated that the precise mechanism for small rather than large vessel disease is unclear.
Hypertensives with abnormal $A B I$ had worse RUDAS score than controls, and in cases, serum HGF concentration was positively associated with RUDAS score, only after further adjustment for SBP and $\mathrm{ABI}$. These findings suggest the negative impact of hypertension and atherosclerosis on the positive association between HGF serum concentration and cognitive function. After further adjustment for lipid profile, the significant association between HGF and RUDAS score was found only in controls. Although of the presence of traditional knowledge about the deleterious effect of lipids upon cognition ${ }^{38}$, this is not a well established risk in recent intervention studies ${ }^{39}$,even some studies linked higher levels of cholesterol, triglyceride and LDL in older adults to a lower risk for vascular dementia and better cognitive performance ${ }^{40-42}$.

\section{CONCLUSIONS}

The potential beneficial link between serum HGF concentration and cognitive function could be met in normotensive subjects with normal ABI. However, this link is halted in the presence of hypertension or atherosclerosis, as assessed by $\mathrm{ABI}$, which was negatively associated with serum HGF concentration. Alternatively, the raised HGF serum level may be an epiphenomenon of atherosclerosis.

\section{References}

1 Nakamura Y, Morishita R, Nakamura S, et al. A novel vascular modulator, hepatocyte growth factor, is associated with systolic pressure. Hypertension 1996;28:409-13.

2 Sato T, Yoshinouchi T, Sakamoto T, et al. Hepatocyte growth factor (HGF): a new biochemical marker for acute myocardial infarction. Heart Vessels 1997;12:241-6.

3 Yamamoto K, Morishita R, Hayashi S, et al. Contribution of Bcl-2, but not Bcl-xL and Bax, to antiapoptotic actions of hepatocyte growth factor in hypoxia-conditioned human endothelial cells. Hypertension 2001;37:1341-8.

4 Taher TEl, Derksen PWB, de Boer OJ, et al. Hepatocyte growth factor triggers signaling cascades mediating vascular smooth muscle cell migration. Biochem Biophys Res Commun 2002;1:80-6.

5 Yamamoto Y, Kohara K, Tabara Y, et al. Plasma hepatocyte growth factor and the relationship between risk factors and carotid atherosclerosis. Hypertens Res 2002;25:661-7.

6 Criqui MH. Peripheral arterial disease - epidemiological aspects. Vasc Med 2001;6(Suppl):3-7.

7 Wright JW, Harding JW. The brain hepatocyte growth Factor/c-Met receptor system: a new target for the treatment of Alzheimer's disease. J Alzheimers Dis 2015;45:9851000 . 
8 Watanabe $\mathrm{T}$, Yamamoto $\mathrm{H}$, Idei $\mathrm{T}$, et al. Influence of insulin-like growth factor-1 and hepatocyte growth factor on carotid atherosclerosis and cognitive function in the elderly. Dement Geriatr Cogn Disord 2004;18:67-74.

9 Tsuboi Y, Kakimoto K, Nakajima M, et al. Increased hepatocyte growth factor level in cerebrospinal fluid in Alzheimer's disease. Acta Neurol Scand 2003;107:81-6.

10 Lieb W, Safa R, Benjamin EJ, et al. Vascular endothelial growth factor, its soluble receptor, and hepatocyte growth factor: clinical and genetic correlates and association with vascular function. Eur Heart J 2009;30:1121-7.

11 Zhu Y, Hilal S, Chai YL, et al. Serum hepatocyte growth factor is associated with small vessel disease in Alzheimer's dementia. Front Aging Neurosci 2018;10:8.

12 Grinberg LT, Heinsen $\mathrm{H}$. Toward a pathological definition of vascular dementia. J Neurol Sci 2010;299:136-8.

13 Aboyans V, Lacroix P, Criqui $\mathrm{MH}$. Large and small vessels atherosclerosis: similarities and differences. Prog Cardiovasc Dis 2007;50:112-25.

14 Laurent S, Briet M, Boutouyrie P. Large and small artery cross-talk and recent morbidity-mortality trials in hypertension. Hypertension 2009;54:388-92.

15 Bonham PA. Get the LEAD Out: noninvasive assessment for lower extremity arterial disease using ankle brachial index and toe brachial index measurements. JWOCN 2006;33:30-41.

16 Newman AB, Shemanski L, Manolio TA, et al. The Cardiovascular Health Study Group: ankle-arm index as a predictor of cardiovascular disease and mortality in the Cardiovascular Health Study. Arterioscler Thromb Vasc Biol 1999;19:538-45.

17 Kjeldsen SE, Dahlof B, Devereux RB, et al. Effects of losartan on cardiovascular morbidity and mortality in patients with isolated systolic hypertension and left ventricular hypertrophy: a Losartan Intervention for Endpoint Reduction (LIFE) substudy. JAMA 2002;288:1491-8.

$18 \mathrm{WHO}$. Health statistics anf information systems. Personal correspondance. Marybeth Weinberger, UN: 2001 (https:// www.who.int/healthinfo/survey/ageingdefnolder/en).

19 WHO. Aging and health. 5 February 2018 (https://www. who.int/news-room/fact-sheets/detail/ageing-and-health).

20 Nishimura M, Nakano K, Ushiyama M, et al. Increased serum concentration of human hepatocyte growth factor in proliferative diabetic retinopathy. J Clin Endocrinol Metab 1998;83:195-8.

21 Taniguchi T, Tei M, Inada K, et al. Serum concentrations of hepatocyte growth factor in breast cancer patients. Clin Cancer Res 1995;1:1031-4.

22 Price JF, McDowell S, Whiteman MC, et al. Ankle brachial index as a predictor of cognitive impairment in the general population: ten-year follow-up of the Edinburgh Artery Study. J Am Geriatr Soc 2006; 54:763-9.

23 Storey JE, Rowland JT, Basic D, et al. The Rowland Universal Dementia Assessment Scale (RUDAS): a multicultural cognitive assessment scale. Int Psychogeriatr 2004;16:13-31.

24 National heart foundation of Australia. Guidelines for the diagnosis and management of hypertension in adults
2016, Melboumi. National heart foundation of Australia 2016 (cited 2018 March 13) (https://www.heartfoundation.org.au/images/uploads/publications/PRO-167_Hypertension-guideline-2016_WEB.pdf).

25 Hirsch AT, Haskal ZJ, Hertzer NR, et al. ACC/AHA 2005 ACC/AHA 2005 Practice Guidelines for the management of patients with peripheral arterial disease (lower extremity, renal, mesenteric, and abdominal aortic): a collaborative report from the American Association for Vascular Surgery/Society for Vascular Surgery, Society for Cardiovascular Angiography and Interventions, Society for Vascular Medicine and Biology, Society of Interventional Radiology, and the ACC/AHA Task Force on Practice Guidelines (Writing Committee to Develop Guidelines for the Management of Patients With Peripheral Arterial Disease): endorsed by the American Association of Cardiovascular and Pulmonary Rehabilitation; National Heart, Lung, and Blood Institute; Society for Vascular Nursing; TransAtlantic Inter-Society Consensus; and Vascular Disease Foundation. Circulation 2006;113:e463-54.

26 Kim HY. Statistical notes for clinical researchers: assessing normal distribution (2) using skewness and kurtosis. Restor Dent Endod 2013;38:52-4.

27 Yoshitomi Y, Kojima S, Umemoto T, et al. Serum hepatocyte growth factor in patients with peripheral arterial occlusive disease. J Clin Endocrinol Metab 1999; 84:2425-8.

28 Nakamura S, Moriguchi A, Morishita R, et al. A novel vascular modulator, hepatocyte growth factor (HGF), as a potential index of the severity of hypertension. Biochem Biophys Res Commun 1998;242:238-43.

29 Bell EJ, Decker PA, Tsai MY, et al. Hepatocyte growth factor is associated with progression of atherosclerosis: the Multi-Ethnic Study of Atherosclerosis (MESA). Atherosclerosis 2018;272:162-7.

30 Decker PA, Larson NB, Bell EJ, et al. Increased hepatocyte growth factor levels over 2 years are associated with coronary heart disease: the Multi-Ethnic Study of Atherosclerosis (MESA). Am Heart J 2019;213:30-4.

31 Hayashi S, Morishita R, Nakamura S, et al. Potential role of hepatocyte growth factor, a novel angiogenic growth factor, in peripheral arterial disease: downregulation of HGF in response to hypoxia in vascular cells. Circulation 1999;100:॥301-8.

32 Powell RJ, Simons M, Mendelsohn FO, et al. Results of a double-blind, placebo controlled study to assess the safety of intramuscular injection of hepatocyte growth factor plasmid to improve limb perfusion in patients with critical limb ischemia. Circulation 2008;118:58-65.

33 Shigematsu H, Yasuda K, Iwai T, et al. Randomized, double-blind, placebo-controlled clinical trial of hepatocyte growth factor plasmid for critical limb ischemia. Gene Ther 2010;17:1152-61.

34 Ishigaki A, Aoki M, Nagai M, et al. Intrathecal delivery of hepatocyte growth factor from amyotrophic lateral sclerosis onset suppresses disease progression in rat amyotrophic lateral sclerosis model. J. Neuropathol Exp Neurol 2007;66:1037-44. 
35 Date I, Takagi N, Takagi K, et al. Hepatocyte growth factor attenuates cerebral ischemia-induced learning dysfunction. Biochem Biophys Res Commun 2004;319:1152-8.

36 Takeuchi D, Sato N, Shimamura M, et al. Alleviation of Abeta-induced cognitive impairment by ultrasound-mediated gene transfer of HGF in a mouse model. Gene Ther 2008;15:561-71.

37 Kuwabara $\mathrm{Y}$, Ichiya $\mathrm{Y}$, Otsuka M, et al. Cerebrovascular responsiveness to hypercapnia in Alzheimer's dementia and vascular dementia of the Binswanger type. Stroke 1992;23:594-8.

38 Ahmadi-Abhari S, Guzman-Castillo M, Bandosz P, et al. Temporal trend in dementia incidence since 2002 and projections for prevalence in England and Wales to 2040: modelling study. BMJ 2017;358:j2856.
39 Moll van Charante EP. Effectiveness of a 6-year multidomain vascular care intervention to prevent dementia (preDIVA): a cluster-randomised controlled trial. Lancet 2016;388:797-805.

40 Mielke MM, Zandi PP, Sjögren M, et al. High total cholesterol levels in late life associated with a reduced risk of dementia. Neurology 2005;64:1689-95.

41 Yin ZX, Shi XM, Kraus VB, et al. High normal plasma triglycerides are associated with preserved cognitive function in Chinese oldest-old. Age Ageing 2012;41:600-6.

42 Lv YB, Yin ZX, Chei CL, et al. Serum cholesterol levels within the high normal range are associated with better cognitive performance among Chinese elderly. J Nutr Health Aging 2016;20:280-7. 